\title{
Pregnancy Induced Microangiopathy, HELLP or TTP!
}

\author{
Ahmed Amro, MD' ${ }^{1}$, Alaa Gabi, MD², and Zeid J. Khitan, MD'
}

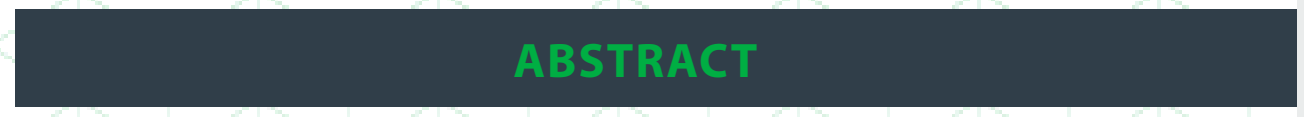

INTRODUCTION: Thrombotic thrombocytopenic purpura (TTP) in pregnancy is rare and can be fatal if misdiagnosed. Typically patients present with microangiopathic hemolytic anemia, thrombocytopenia, altered mental status, fever, and renal abnormalities

CASE DESCRIPTION: We are presenting a case of 26 year old pregnant female presented with elevated BP. She had scattered Bruises in her legs, thighs and arms along with +2 bilateral pitting leg edema. Initial and subsequent laboratory studies showed features of microangiopathic hemolytic anemia (MAHA), which brought up the suspicion of HELLP (hemolysis, elevated liver enzymes, low platelets) syndrome initially. Labor was induced after transfusion of 2 units of PRBCs and 2 units of Platelets. The patient platelets and hemoglobin showed no improvement after delivery and she continued to have hemolysis despite delivery and steroids administration. Daily therapeutic plasma exchange was initiated as the index of suspicion for TTP increased. After two sessions, there was a significant improvement in the platelet count and hemolysis profile which were normalized by the 4 th session.

CONCLUSION: Congenital TTP is a rare syndrome that clinicians should be aware of. Early initiation of plasma exchange dramatically improves survival from less than $10 \%$ to approximately $80 \%$. This can only be achieved by having high level of suspicion and awareness to this possibility.
Author affiliations are listed at the end of this article.

Correspondence to: Zeid J. Khitan, MD Marshall University Joan C. Edwards School of Medicine zkhitan@marshall.edu

\section{KEYWORDS}

TTP, Congenital TTP, Pregnancy, HELLP, Preeclampsia, Upshaw-Schulman syndrome

\section{INTRODUCTION}

Thrombotic thrombocytopenic purpura (TTP) in pregnancy is rare and can be fatal if misdiagnosed. ${ }^{1}$ Hereditary or acquired TTP must be considered in the differential diagnosis of pregnancy-induced microangiopathic hemolytic anemia (MAHA) along with severe preeclampsia as in hemolysis, elevated liver enzymes, low platelets (HELLP) syndrome. ${ }^{2}$ In TTP, early initiation of plasma exchange dramatically improves survival from less than $10 \%$ to approximately $80 \%{ }^{1}$

\section{CASE PRESENTATION}

A 26 year old Caucasian female presented for a routine peripartum visit. She was G1P0 at 35 weeks gestation. Her pre-partum history was significant for mild bruising tendency since childhood and subnephrotic proteinuria.

She was found to have elevated blood pressure of 140/90 and had 13 lbs. weight gain over a two week period. She reported increasing swelling in her legs and face one week prior to presentation. She denied headaches, blurry vision, right upper quadrant pain, dyspnea, nausea, or vomiting. Other than 
hypertension, physical exam showed vital signs of HR 88 RR 18 and Temp of 98. Abdomen was soft, gravid, and non-tender. She had scattered bruises in her legs, thighs and arms along with +2 bilateral pitting leg edema. Initial and subsequent laboratory studies are shown in the table.

The combination of high blood pressure, proteinuria, hemolytic anemia and thrombocytopenia brought up the suspicion of HELLP syndrome. Labor was induced after transfusion of 2 units of PRBCs and 2 units of platelets. The mother and her newborn were closely observed on labor and delivery unit. The fetal heart rate showed no major abnormalities. Apgar score was $3 \& 8$ at $1 \& 5$ minutes respectively. The patient platelets and hemoglobin showed no improvement and she continued to have hemolysis despite delivery and steroids administration. At this point, daily therapeutic plasma exchange was initiated as the index of suspicion for TTP increased. After two sessions, there was a significant improvement in the platelet count and hemolysis profile which were normalized by the 4th session.

\section{DISCUSSION}

TTP (Moschcowitz syndrome) is an acute, rare,

TABLE: The patient laboratory work up

\begin{tabular}{|c|c|c|}
\hline Test & Result & Reference Range \\
\hline Hemoglobin & $7.2 \mathrm{~g} / \mathrm{dl}$ & $12.0-16.0$ \\
\hline Platelets & 16 & $15-440$ \\
\hline WBC & 9.2 & $4.5-10.0$ \\
\hline Sodium & 141 & $135-145$ \\
\hline Potassium & 4.1 & $3.5-5.0$ \\
\hline Calcium & 8.2 & $8.5-10.5$ \\
\hline Creatinine & 0.78 & $0.6-1.10$ \\
\hline Blood Urea Nitrogen & 16 & $7-21$ \\
\hline Glucose & 95 & $65-110$ \\
\hline Albumin & 3.2 & $3.5-5.0$ \\
\hline Total Bilirubin & 0.6 & \begin{tabular}{|l|}
$0.3-1.30$ \\
\end{tabular} \\
\hline AST & 31 & $10-40$ \\
\hline ALT & 21 & $7-40$ \\
\hline GGT & 9 & $12-58$ \\
\hline PT & 9.9 & \begin{tabular}{|l|}
$9.4-12.4$ \\
\end{tabular} \\
\hline aPTT & 25.5 & 20.41-33.39 \\
\hline INR & 0.92 & \begin{tabular}{|l|}
$0.90-1.30$ \\
\end{tabular} \\
\hline Urine Creatinine & 265 & $20-320$ \\
\hline Urine Protein & 870 & $40-80$ \\
\hline Urinalysis & Moderate amount of intact RBC & 0-1 RBC \\
\hline Urine drug screen & \begin{tabular}{|l|} 
negative \\
\end{tabular} & \begin{tabular}{|l|} 
Negative \\
\end{tabular} \\
\hline ANA & negative & Negative $(<1: 80)$ \\
\hline HIV & \begin{tabular}{|l|} 
negative \\
\end{tabular} & \begin{tabular}{|l|} 
Negative \\
\end{tabular} \\
\hline Hepatitis Panel & negative & Negative \\
\hline Blood group & $\mathrm{O}^{+}$ & \\
\hline Peripheral Blood Smear & $\begin{array}{l}\begin{array}{l}\text { Schistocytes, Microspherocytes } \\
\text { and Toxic PMNs }\end{array} \\
\end{array}$ & \\
\hline Direct Coombs Test & \begin{tabular}{|l|} 
negative \\
\end{tabular} & \begin{tabular}{|l|} 
Negative \\
\end{tabular} \\
\hline Reticulocyte Count & 9.88 & 0.73-3.12 \\
\hline Haptoglobin & Less than 7 & $32-154$ \\
\hline Fibrinogen & 493.5 & $140.0-498.0$ \\
\hline LDH & 473 & \begin{tabular}{|l|}
$100-190$ \\
\end{tabular} \\
\hline Uric Acid & 7.1 & \begin{tabular}{|l|}
$2.5-7.5$ \\
\end{tabular} \\
\hline Anticardiolipin & Negative $(<9)$ & Negative (0-14) \\
\hline B2 Glycoprotein 1 Antibodies & Negative $(<9)$ & Negative $(0-20)$ \\
\hline ADAMTS13 Activity & Less than $10 \%$ & $>66 \%$ \\
\hline ADAMTS13 Antibody & Negative $(<9)$ & Negative $(<12)$ \\
\hline Placenta Pathology & $\begin{array}{l}\text { Intraparenchymal infarct, } \\
\text { Perivillous and Perivascular } \\
\text { fibrin deposition }\end{array}$ & \\
\hline
\end{tabular}
and potentially fatal disorder. ${ }^{3}$ The classical pentad of clinical features includes microangiopathic hemolytic anemia, thrombocytopenia, neurologic symptoms, fever, and renal abnormalities. ${ }^{4}$ It is caused by absent or severely depleted ADAMTS-13, the von Willebrand factor (VWF) cleaving protease. 3 It is either acquired, likely immune mediated, or hereditary, also called Upshaw-Schulman syndrome. ${ }^{5}$

Upshaw-Schulman syndrome or Congenital TTP (cTTP) is caused by homozygous or compound heterozygous ADAMTS13 mutations. ${ }^{6-7}$ It is diagnosed by the documentation of a severe deficiency in ADAMTS13 activity $(<10 \%)$, and absence of ADAMTS13 autoantibody. The diagnosis is confirmed by documentation of ADAMTS13 mutation. ${ }^{8}$ cTTP clinical signs may be mild or absent during childhood. History of easy bruising during childhood due to isolated thrombocytopenia can be the only symptom. ${ }^{9}$ There are two age clusters for the initial episode of CTTP; about half of patients have their first acute episode in infancy or early childhood, while the other half remain asymptomatic 
until adulthood, ${ }^{9}$ with pregnancy being the most important precipitant of initial acute episode. ${ }^{7}$ Physiologic changes during pregnancy including progressive increase of VWF and associated decrease of ADAMTS13 can be considered a likely triggering mechanism for the acute episodes of TTP.5 Pregnancy induced cTTP mostly occurs in the third trimester, ${ }^{3,9}$ which is likely related to the period over which the level of VWF is increased during pregnancy. ${ }^{5,9}$

On the other hand, preeclampsia/HELLP syndrome can be associated with thrombocytopenia, microangiopathic hemolytic anemia, neurologic symptoms, and renal insufficiency, making their distinction from TTP difficult or impossible especially since their onset can be delayed to the third trimester of pregnancy as in TTP. ${ }^{3,9}$ There are some features that may favor the diagnosis of TTP over HELLP syndrome which include severe thrombocytopenia (less the 20,000) and an elevated LDH-to-AST ratio. ${ }^{3}$ ADAMTS-13 activity might be reduced along with elevated VWF levels in HELLP syndrome, but a severe reduction in ADAMTS-13 activity $(<10 \%) 10$ along with an increase in ultra large VWF multimers is usually suggestive of TTP. ${ }^{3}$ On the other hand HELLP syndrome is usually associated with preeclampsia, so hypertension and proteinuria would favor a diagnosis of HELLP syndrome.

The probability of serious maternal and fetal complications during pregnancy is high in patients with cTTP. ${ }^{5}$ Untreated TTP is associated with a $>90 \%$ mortality. ${ }^{1}$ Without appropriate treatment, the risk of recurrent episode in patients with cTTP during the next pregnancy is almost $100 \% .5$ Daily plasma exchange and glucocorticoids is the main therapy for adult patients with TTP during pregnancy, ${ }^{4}$ which dramatically improved survival from less than $10 \%$ to approximately $80 \% .{ }^{1,3}$ Patients with CTTP respond quickly to plasma transfusion either upon need or intermittently every 2-3 weeks as a prophylactic therapy. Some plasma derived factor VIII/VWF concentrates contain sufficient amount of ADAMTS13 that was found to be useful in treating CTTP, replacing FFP and plasma exchange. ${ }^{11}$ CONCLUSION

Congenital TTP is a rare syndrome that clinicians should be aware of. It can be misdiagnosed in pregnancy as HELLP syndrome, which is more common in this group of patients. If untreated, death from disseminated microvascular thrombosis occurs which makes early detection and treatment of this disease life saving. This can only be achieved by having high level of suspicion and awareness to this possibility.

\section{AUTHOR AFFILIATIONS}

1. Department of Internal Medicine, Marshall University Joan C. Edwards School of Medicine.

2. Department of Cardiovascular Services, Marshall University Joan C. Edwards School of Medicine.

\section{REFERENCES}

1. McMinn JR, George JN. Evaluation of women with clinically suspected thrombotic thrombocytopenic purpura-hemolytic uremic syndrome during pregnancy. J clin apheresis. 2001;16(4):202-9.

2. Weinstein L. Syndrome of hemolysis, elevated liver enzymes, and low platelet count: a severe consequence of hypertension in pregnancy. Am J Obstet Gynecol. 1982;142(2):159-67.

3. Fyfe-Brown A, Clarke G, Nerenberg K, Chandra $S$, Jain V. Management of pregnancy-associated thrombotic thrombocytopenia purpura. AJP reports. 2013;3(1):45-50.

4. Lucania G, Camiolo E, Carmina MG, Fiandaca $T$, Indovina A, Malato A, et al. Multidisciplinary approach in pregnancy-associated thrombotic thrombocytopenic purpura: a case report. Blood transfusion = Trasfusione del sangue. 2014;12 Suppl 1:s137-40.

5. Falter T, Kremer Hovinga JA, Lackner K, Fullemann HG, Lammle B, Scharrer I. Late onset and pregnancy-induced congenital thrombotic thrombocytopenic purpura. Hamostaseologie. 2014;34(3):244-8.

6. George JN, Nester CM. Syndromes of thrombotic microangiopathy. N Engl J Med. 2014;371(7):65466.

7. Fujimura $Y$, Matsumoto $M$, Kokame $K$, Isonishi A, Soejima K, Akiyama N, et al. Pregnancy-induced thrombocytopenia and TTP, and the risk of fetal 
death, in Upshaw-Schulman syndrome: a series of 15 pregnancies in 9 genotyped patients. $\mathrm{Br} \mathrm{J}$ Haematol. 2009;144(5):742-54.

8. Scully $M$, Thomas $M$, Underwood $M$, Watson $\mathrm{H}$, Langley K, Camilleri RS, et al. Thrombotic thrombocytopenic purpura and pregnancy: presentation, management, and subsequent pregnancy outcomes. Blood. 2014;124(2):211-9.

9. George JN. The association of pregnancy with thrombotic thrombocytopenic purpurahemolytic uremic syndrome. Curr Opin Hematol. 2003;10(5):339-44.

10. Jiang Y, Mclntosh JJ, Reese JA, Deford CC, Kremer Hovinga JA, Lammle B, et al. Pregnancy outcomes following recovery from acquired thrombotic thrombocytopenic purpura. Blood. 2014;123(11):1674-80.

11. Rosove MH. Thrombotic microangiopathies. Semin Arthritis Rheum. 2014;43(6):797-805. 